\title{
Wolbachia modification of sperm does not always require residence within developing sperm
}

\author{
ME Clark ${ }^{1}$, C Bailey-Jourdain ${ }^{2}$, PM Ferree $^{3}$, SJ England $^{1}$, W Sullivan ${ }^{3}$, DM Windsor ${ }^{4}$ and JH Werren ${ }^{1}$ \\ ${ }^{1}$ Department of Biology, University of Rochester, Rochester, NY, USA; ${ }^{2}$ Department of Natural Resource Sciences, McGill University, \\ Montreal, Canada; ${ }^{3}$ Department of Molecular, Cellular and Developmental Biology, 319 Sinsheimer Laboratories, University of \\ California, Santa Cruz, CA, USA and ${ }^{4}$ Smithsonian Tropical Research Institute, Apartado 2072, Balboa, Republic of Panama
}

\begin{abstract}
Wolbachia are maternally inherited intracellular bacteria known to manipulate the reproduction of their arthropod hosts. Wolbachia commonly affect the sperm of infected arthropods. Wolbachia-modified sperm cannot successfully fertilize unless the female is infected with the same Wolbachia type. A study of spermatogenesis in the parasitic wasp Nasonia vitripennis reveals that Wolbachia are not required in individual spermatocytes or spermatids to modify sperm. In N. vitripennis, Wolbachia modify nearly all sperm, but are found only in $\sim 28 \%$ of developing sperm, and are also found in surrounding cyst and sheath cells. In
\end{abstract}

the beetle Chelymorpha alternans, Wolbachia can modify up to $90 \%$ of sperm, but were never observed within the developing sperm or within the surrounding cyst cells; they were abundant within the outer testis sheath. We conclude that the residence within a developing sperm is not a prerequisite for Wolbachia-induced sperm modification, suggesting that Wolbachia modification of sperm may occur across multiple tissue membranes or act upstream of spermiogenesis.

Heredity (2008) 101, 420-428; doi:10.1038/hdy.2008.71; published online 23 July 2008

Keywords: Wolbachia; spermatogenesis; cytoplasmic incompatibility; Nasonia vitripennis; Chelymorpha alternans

\section{Introduction}

Wolbachia are among the most common of endosymbiotic bacteria found in a large proportion of arthropods and filarial nematodes (Bandi et al., 1998; Windsor and Werren, 2000). Most surveys suggest that at least $20 \%$ of terrestrial arthropods are infected with Wolbachia (Werren et al., 1995; West et al., 1998; Jeyaprakash and Hoy, 2000; Windsor and Werren, 2000; Sintupachee et al., 2006). A recent meta-analysis of Wolbachia surveys estimates that $66 \%$ of insect species harbor Wolbachia (Hilgenboecker et al., 2008).

Wolbachia are maternally (cytoplasmically) inherited bacteria and Wolbachia-induced manipulation of host reproduction results in increased proportion of infected females in a population (Caspari and Watson, 1959; Werren and O'Neill, 1997). Among the most common manipulations are host feminization, parthenogenesis induction, male killing and cytoplasmic incompatibility (CI) (Werren, 1997; Stouthamer et al., 1999) as well as other, sometimes subtle, effects on hosts (Clark, 2007).

Cytoplasmic incompatibility is a form of conditional infertility where sperm from a Wolbachia-infected male fertilize eggs but development fails unless the eggs are infected with the same Wolbachia type(s). CI is the most commonly described Wolbachia-induced phenotype, documented in at least eight different arthropod orders

Correspondence: Dr ME Clark, Department of Biology, University of Rochester, Hutchison Hall, Rochester, NY 14627, USA.

E-mail:mclark11@mail.rochester.edu

Received 12 February 2008; revised 27 May 2008; accepted 20 June 2008; published online 23 July 2008 including Acari (Breeuwer and Jacobs, 1996), Coleoptera (Wade and Stevens, 1985), Diptera (Yen, 1975), Homoptera (Hoshizaki and Shimada, 1995), Hymenoptera (Reed and Werren, 1995), Isopoda (Moret et al., 2001), Lepidoptera (Brower, 1976) and Orthoptera (Kamoda et al., 2000).

There are at least two distinct events in CI, the Wolbachia-induced modification of sperm and the Wolbachia-induced rescue of that modification upon fertilization (Werren, 1997). Following fertilization with a Wolbachia-modified sperm, the result is either (1) normal development in embryos from eggs harboring at least the same Wolbachia types as the father or (2) abnormal development in embryos lacking the father's Wolbachia type(s). Incompatibility is manifested as a disruption of pronuclear chromatin condensation followed by missegregation of chromosomes during mitosis (Reed and Werren, 1995; Callaini et al., 1996).

The molecular mechanisms underlying CI are currently unknown, as is the specific stage(s) of spermatogenesis during which Wolbachia modify the developing sperm. Most of which is currently known regarding Wolbachia during spermatogenesis comes from studies on Drosophila. Wolbachia are present in a subset of cysts throughout spermatogenesis and removed from spermatids during individualization along with most of the cytoplasm and the minor mitochondrial derivative. Wolbachia are not found in mature sperm (Bressac and Rousset, 1993; Snook et al., 2000; Clark et al., 2002). Studies of CI in Drosophila suggested that Wolbachia are required within developing spermatocytes and spermatids for those sperm to be modified, because rates of CI (percentage of embryos affected in an incompatible 
cross) correlate with the proportion of developing cysts with Wolbachia within spermatocytes and spermatids (Clark et al., 2003; Veneti et al., 2003). However, it should be noted that the conclusion is based solely on this correlation, and not on functional studies demonstrating a requirement of Wolbachia to be present in individual cysts to affect modification.

So far, studies of Wolbachia during spermatogenesis have only been conducted in Drosophila, which could lead to inaccurate generalizations for other host species. Here, we investigate the patterns of Wolbachia infection during spermatogenesis in the parasitic wasp Nasonia vitripennis and the beetle Chelymorpha alternans from two additional insect orders. $N$. vitripennis has been a model for Wolbachia studies for many years. Levels of CI are $97-100 \%$, and the species is stably infected with two different Wolbachia strains, $w \mathrm{NvA} 1$ and $w \mathrm{NvB} 1$ (Bordenstein and Werren, 1998; Bordenstein et al., 2003). In N. vitripennis, CI results in total elimination of the paternal chromosomes, converting a diploid embryo, which would have developed into a female, into a haploid male in this haplo-diploid species (Reed and Werren, 1995). C. alternans shows high levels of CI (up to $90 \%$ when doubly infected) with two infection forms routinely found in nature, singly infected insects with strain $w$ Calt1 and doubly infected insects with $w$ Calt1 and wCalt2 (Keller et al., 2004). Double-infected males are incompatible with single-infected females. In both species, cured strains are available to measure the levels of CI. Our results indicate that, contrary to conclusions of previous studies in Drosophila, Wolbachia are not required in developing sperm of $N$. vitripennis and C. alterans to induce modification.

\section{Materials and methods}

\section{Stocks}

$N$. vitripennis: $\mathrm{R} 511$ is a doubly infected line with Wolbachia from both the A and B supergroups (PerrotMinnot et al., 1996). R511-T is an uninfected line, created from R511 by two generations of tetracycline treatment, as described in Breewuer and Werren (Breeuwer and Werren, 1990). AsymC is an N. vitripennis line previously cured of Wolbachia (Breeuwer and Werren, 1990). All wasps were reared at $25^{\circ} \mathrm{C}$ under constant light on Wolbachia-free Sarcophaga bullata pupa (fleshfly) in uncrowded conditions.

C. alternans: Three different lines of C. alternans males, doubly infected (Gamboa), singly infected (Guarumal) and uninfected (Gamboa) were examined. Uninfected stocks of C. alternans were created by injecting females from Gamboa three times a week with a solution of 2-3 $\mu 1$ of sterile $0.9 \%$ rifiampicin for two consecutive weeks as described previously (Keller et al., 2004). Amplification of the $952 \mathrm{bp} 16 \mathrm{~S}$ rDNA gene fragment using general primers and the $560 \mathrm{bp} w s p$ gene fragment using Wolbachia strain-specific primers were used to validate the negative infection status of the treated lines (Keller et al., 2004). All C. alternans were kept in the laboratory (12 h light, $60 \%$ humidity, $26^{\circ} \mathrm{C}$ temperature) on fresh leaves of Merremia umbellata (Convolvulaceae). Egg masses were placed in a percival I-30BL incubator adjusted to give a $13-11 \mathrm{~h}$ dark-light cycle, $26-28^{\circ} \mathrm{C}$ temperature and 70-75\% humidity from day 0 until hatching and then reared under the laboratory conditions described above.

\section{$\mathrm{Cl}$ measurements}

Cytoplasmic incompatibility is estimated in both Nasonia and Chelymorpha using long established standard methods. In N. vitripennis, CI was estimated by comparing the proportion of females in an incompatible cross (IC: infected male $\times$ uninfected female) divided by the percentage of female progeny in the compatible cross using the same strain females (CC: uninfected male $x$ uninfected female) according to the following index $\mathrm{CI}=1-$ (percentage of female $\mathrm{IC} /$ percentage of female $\mathrm{CC}) \times 100$. This assay takes into consideration that female progeny are derived normally from fertilized eggs in this haplodiplod insect and CI results in paternal chromosome loss and conversion to haploid males in this species (Reed and Werren, 1995; Bordenstein et al., 2003). Mortality from egg laying to adulthood is a minor contributor under the assay conditions used here (Bordenstein et al., 2003). In C. alternans, CI was estimated by comparing egg hatch rates in control versus incompatible crosses, using the following index $\mathrm{CI}=(\mathrm{IUE}-\mathrm{CUE}) /(1-\mathrm{CUE}) \times 100$, where IUE represents the percentage of unhatched eggs observed in incompatible cross and CUE represents the percentage of unhatched eggs in compatible crosses of the tested strain (Poinsot et al., 1998). The average CUE used here was 28\% (Bailey-Jourdain, 2006).

\section{Cytology}

Nasonia testes were dissected from pupa and adults in TBST (50 mM Tris, $150 \mathrm{mM} \mathrm{NaCl}, 0.1 \%$ Tween, $0.05 \%$ $\mathrm{NaN}_{3}, \mathrm{pH}$ 7.5). They were then either transferred whole into a microcentrifuge tube or transferred to a lysinecoated slide for further dissection. Tissues were fixed in $3.7 \%$ formaldehyde in TBST for $15-30 \mathrm{~min}$, followed by three washes in TBST for 5 min each and blocked in TBST with $1 \%$ bovine serum albumin (BSA) for $10 \mathrm{~min}$. Wolbachia were visualized using a rabbit polyclonal antibody made against a portion of the Wolbachia surface protein (wsp; provided by $\mathrm{K}$ Bourtzis). Tissues were incubated in the primary antibody solution (TBST, 1\% BSA, $2 \mathrm{mg} \mathrm{ml}^{-1}$ RNaseA and 1:500 of the anti-wsp antibody), for $1 \mathrm{~h}$ at room temperature, followed by three washes with TBST. This was followed by $1 \mathrm{~h}$ at room temperature in 1:500 alexa-flour 488 antirabbit antibody (Invitrogen, Carlsbad, CA, USA), followed by three washes in TBST. DNA was then stained with $5 \mu \mathrm{g} \mathrm{ml}^{-1}$ propidium iodide for $20 \mathrm{~min}$, followed by a brief wash in TBST before mounting in ProLong Gold antifade mounting media (Invitrogen). Confocal images were obtained using a Leica SP confocal microsope.

To determine the proportion of developing sperm infected, testes from 10 males were dissected from the yellow pupal stage (just before the beginning of meiosis) (Pultz and Leaf, 2003). Cysts were removed and further disrupted by brief pipetting. The resulting tissue was deposited on a lysine-coated slide, fixed and stained as above. The result was large numbers spermatocytes either singly or in groups of small numbers. Spermatocytes were scored for the presence of Wolbachia. 
Chelymorpha testes from 4-week-old singly infected (Guarumal), doubly infected (Gamboa) and uninfected (Gamboa) beetles were examined. This is shortly after males reach sexual maturity (D Windsor, unpublished). At this stage, CI levels of Gamboa males crossed to uninfected females is $75.9 \%$ and Guarumal males crossed to uninfected females is $67.2 \%$, respectively (BaileyJourdain, 2006). Testes were removed and fixed for $20 \mathrm{~min}$ in $100 \mu \mathrm{l}$ of $3.7 \%$ formaldehyde in phosphate buffer and $600 \mu \mathrm{l}$ of heptane. Samples were then rinsed three times for $10 \mathrm{~min}$ in PSBT (phosphate buffer $+0.3 \%$ Triton X-100) and DNA was stained with either $4^{\prime}, 6-$ diamidino-2-phenylindole (DAPI) $\left(1.0 \mu \mathrm{g} \mathrm{ml}^{-1}\right)$ for $5 \mathrm{~min}$ or Oligreen (1:750) for $20 \mathrm{~min}$ and rhodamine-labeled phalloidin (1:100) for $90 \mathrm{~min}$. The phalloidin labels host actin, allowing for visualization of different cell layers and differentiating cysts from the outer testis sheath. Images were obtained using a Leica DM IRB confocal microscope and a Nikon E600. To confirm the results from visualization of Wolbachia in whole testes, fixed testes were further dissected and cysts removed and examined.

\section{Sperm depletion}

Rates of sperm utilization in Wolbachia infected and uninfected $N$. vitripennis males were estimated by comparing rates of sperm depletion upon successive matings. Wolbachia infected and uninfected males were repeatedly mated to infected females. The relative rates of sperm depletion were estimated by the proportion of female offspring (fertilized eggs) resulting from those matings. All females used were Wolbachia infected, eliminating the confounding effects of Wolbachia-induced incompatibility.

Over four $24 \mathrm{~h}$ periods, both infected (R511) and uninfected (R511-T) males were presented with 24 females with which to mate, eight females sequentially for $1 \mathrm{~h}$ each and 16 females in mass overnight. For each of the eight, $1 \mathrm{~h}$ parings per day, the pair was observed until copulation was seen. Following mating, each female was given two hosts for $48 \mathrm{~h}$ and then removed. Offspring were counted for three of the eight, $1 \mathrm{~h}$ matings.

\section{Mating history}

Wolbachia infected N. vitripennis males (R511) were repeatedly mated and periodically tested for CI. Males were alternately presented infected and uninfected females for $1 \mathrm{~h}$ each. Pairs were observed until mating was seen. The uninfected females were used to assay CI, the infected females were to determine whether sperm was still being transferred. Uninfected males (AsymC) were similarly treated. A total of five males of each type were used. Data presented as total sum of offspring by sex.

\section{Results}

\section{Nasonia $\mathrm{Cl}$ and spermatogenesis}

Nasonia normally give rise to both fertilized (diploid) and unfertilized (haploid) offspring. CI is measured by the effect on offspring derived from fertilized eggs in an incompatible cross. In N. vitripennis, the result of CI is loss of paternal chromosomes and resulting haploid (male) development (Reed and Werren, 1995; Tram et al.,
2006). In an incompatible cross (infected male $x$ uninfected female), the result is an increase in male offspring. Consistent with previous results (PerrotMinnot et al. 1996), the doubly infected males used in these experiments yielded nearly all male offspring $(97.96 \% \pm 0.11$ s.e.m., $n=19)$ when mated to uninfected females.

Spermatogenesis in Nasonia shares many features with the well-characterized events in Drosophila (Fuller, 1993). Each male has two testes, composed of a single follicle. Within testes, sperm develop within cysts composed of two somatic cells (cyst cells) surrounding a number of interconnected germline cells (spermatocytes/spermatids). The two cyst cells form an envelope completely surrounding the germline component of the cyst, the sheath cells. With cyst development, the two cyst cells enlarge to accommodate the growing cyst, but do not further divide. Within the cyst interior, the germline spermatocyte undergoes several rounds of mitotic division, followed by meiosis. As male Nasonia are normally haploid, meiosis is composed of an aborted meiosis I, followed by normal meiosis II (Pennypacker, 1958) (Figure 1a). A notable and potentially important difference in spermatogenesis between Nasonia and Drosophila is the variation in the stages of development found within a testis. Spermatogenesis is largely synchronized in Nasonia (Pennypacker, 1958), probably due to the fact that most matings occur immediately after male eclosion (Whiting, 1967). Most of the cysts within an N. vitripennis testis are therefore of the same or similar developmental stage in maturing pupae (Figure 1b). Additionally, the cyst cells within a Nasonia testis contain giant polyploid nuclei (Figures $1 \mathrm{a}$ and $\mathrm{g}$ ). The implications of these differences on the expression of CI are discussed below.

Wolbachia can be seen throughout the whole testis in $N$. vitripennis (Figure 1b). The exact localization of Wolbachia, however, is not evident in the initial observation, necessitating closer examination of individual developing cysts. In addition to the Wolbachia found within the outer testis sheath, Wolbachia were frequently seen within both germline (spermatocytes/spermatids) and soma (cyst cells) within individual cysts, but at densities much lower than normally observed in Drosophila melanogaster (Clark et al., 2002). Hundreds of cysts from many different testes were examined. Figure 1 shows some of the characteristic cyst infections. Wolbachia levels within cysts increases with cyst development (compare Figures 1c-h). Although the level of Wolbachia within cysts was highly variable, Wolbachia were seen in nearly every developing cyst. Accurately scoring the infection status of individual whole cysts was unattainable because most cysts removed from a testis do not remain intact, especially cyst cells. In contrast to cysts, Wolbachia were not found within the majority of developing spermatocytes and spermatids. Specifically, scoring of infections in premeiotic spermatocytes shows that only $28 \%$ $(N>1500)$ of spermatocytes at this stage were infected with Wolbachia. Although impossible to quantify later in development due to the difficulty in separating bundles of spermatids, this infection frequency in spermatocytes is consistent with that observed in more developed spermatids (Figure 1). Therefore, only a minority of developing sperm is infected with Wolbachia. However, based on complete CI in crosses between infected males and uninfected females, compared to the control infected 
a
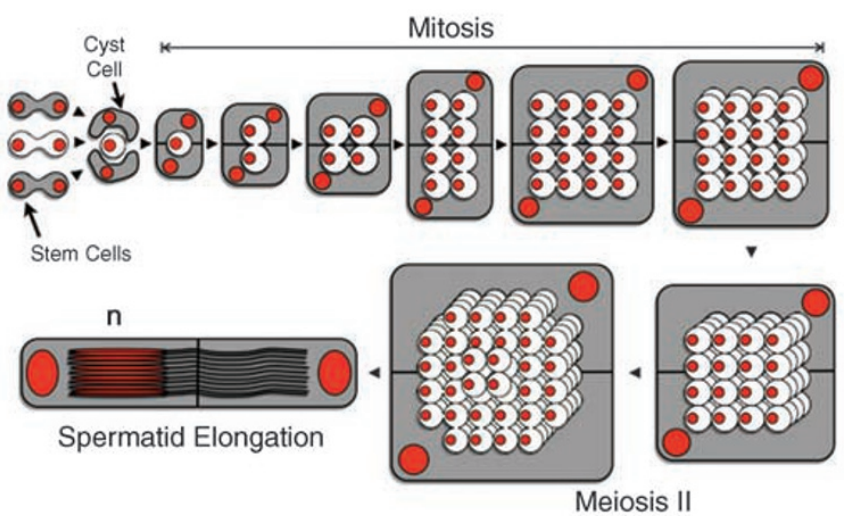
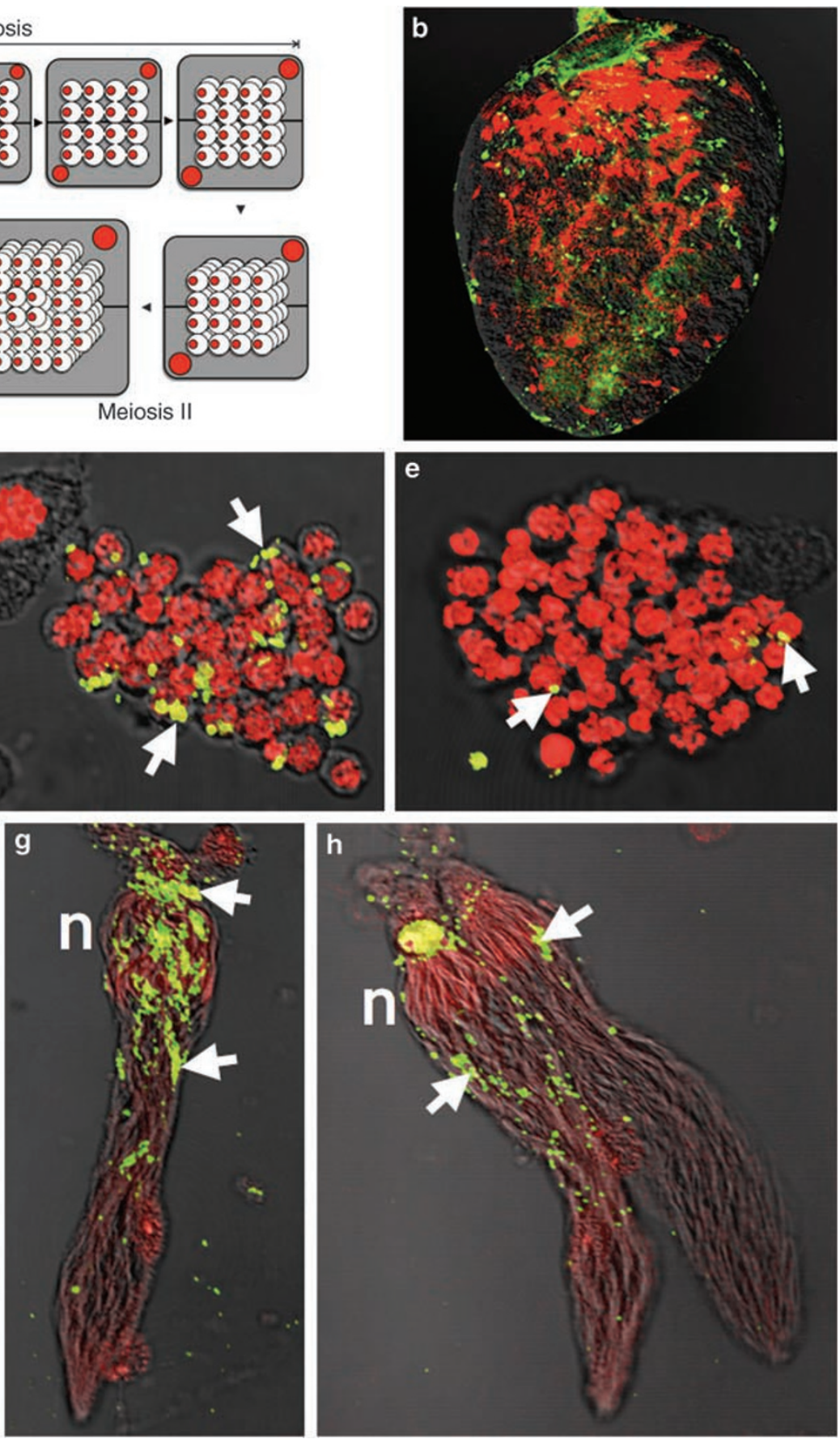

Figure 1 Wolbachia and spermatogenesis in N. vitripennis. (a) Schematic diagram of spermatogenesis in Nasonia. Spermatogenesis begins with a spermatogonial stem cell dividing and forming a daughter primary gonial cell. Cyst progenitor (stem) cells are divided to form cyst cells, two of which surround a primary gonial cell. The primary gonial cell undergoes several rounds of mitosis, aborted meiosis I and normal meiosis II. The haploid spermatids then elongate with the growth of the sperm tails. The germ cells are shown in white, somatic cells are shown in gray. (b) Wolbachia (green/yellow) within a whole testis from a late stage pupa. Clusters of red nuclei are cysts in the latter stages of development. (c-e) Wolbachia within premeiotic spermatocysts. (f-h) Wolbachia within elongated spermatocysts. Arrows indicate Wolbachia. $\mathrm{n}=$ spermatid nuclei. See online version for colour figure.

male $\times$ infected female and uninfected male $\times$ infected female crosses, nearly $100 \%$ of sperm are modified. In this study, CI was estimated at $97.96 \%$ consistent with previous reports of complete or near complete CI (PerrotMinnot et al., 1996). Yet only around $28 \%$ of developing spermatocytes carry Wolbachia, suggesting that the bacteria do not need to be in the developing spermatocytes and spermatids to induce sperm modification.
An alternative explanation is that Wolbachia only modify those developing sperm, which harbor Wolbachia during development, and these sperm are used exclusively or disproportionately for fertilization, whereas the majority of sperm from uninfected spermatocytes are unutilized or nonfunctional. If the latter were true, and Wolbachia infected males utilize only those sperm infected during development, then infected males 

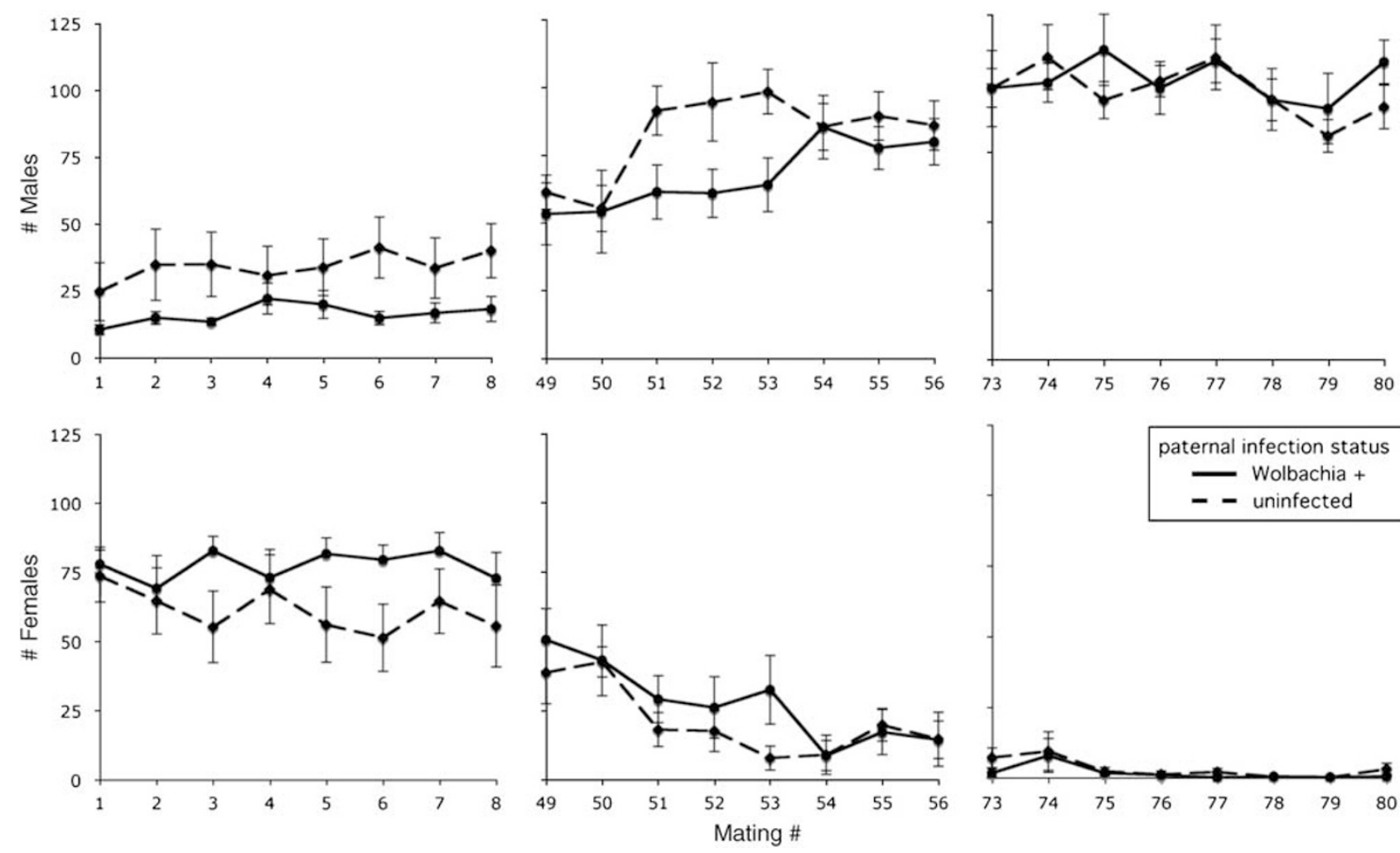

Figure 2 The effect of paternal infection status and successive mating on the number of male and female progeny. All females were Wolbachia infected, eliminating cytoplasmic incompatibility. Error bars indicate standard errors.

should suffer a great reduction in fertility compared to uninfected males. To distinguish between these possibilities, fertility of infected and uninfected males was compared by repeatedly mating to infected females. Fertility (total usable sperm) was estimated by counting the numbers of female offspring resulting from each mating. Each of the 10 infected males tested lived long enough to be presented with at least 80 females (one uninfected male died between female numbers 78 and 79; Figure 2). The surviving males continued to copulate with each female observed. For each mating examined (mating numbers $1-8,49-56$ and $73-80$ ), there was no significant difference in the number of female offspring (Mann-Whitney $U$-test, $P>0.05$ ). There was a significant difference in the number of male offspring in only three of the 24 matings examined number $6, P=0.0412$; number $8, P=0.0411$ and number $53, P=0.0211$ ). In each of these three crosses, there were more male offspring from females mated to uninfected versus infected males. By mating number 75, both infected and uninfected males sired few or no female offspring, suggesting that sperm was completely or nearly depleted at that time. In addition to the three crosses with significant differences in the number of male offspring, there was a consistent trend (although not statistically significant at any single time period) toward fewer female offspring from females mated to uninfected versus infected males, the opposite trend to that expected if infected males produced large numbers of nonfunctional sperm. These results argue against the hypothesis that uninfected spermatocytes from infected males are nonfunctional.

Furthermore, no reduction in CI was observed regardless of numbers of previous matings. Among 84 different pairings, infected males were assayed for $\mathrm{CI}$ at 13 different times. The result was always $100 \%$ male offspring. Therefore, CI in Nasonia is not reduced with increased numbers of male mating (Figure 3).

\section{Chelymorpha $\mathrm{Cl}$ and spermatogenesis}

Cytoplasmic incompatibility levels were examined for both Gamboa double-infected males and Guaramal single-infected males crossed to uninfected Gamboa females, at male age of 4 weeks. After CI measurement, the males were used for cytological examinations. Gamboa males crossed to uninfected females showed $75.9 \%$ incompatibility ( $N=5$, range: $63.4-90.5 \%)$, indicating that approximately $75.8 \%$ of the sperm were sufficiently modified to cause CI. These same males were dissected upon mating and used for cytological examinations. Guarumal males crossed to uninfected females gave incompatibility of $67.2 \%(N=6$, range: 33.3-87.4\%).

As in Nasonia and Drosophila, the Chelymorpha testis is composed of developing cysts surrounded by an outer epithelial sheath cells. The cyst consists of two cell types, the germ cells (spermatocytes that develop into spermatids) surrounded by two somatic cyst cells. Each region was examined for Wolbachia. In contrast to Drosophila and Nasonia, spermatogenesis in Chelymorpha occurs within two disc-shaped testes composed of multiple follicles. Each follicle is homologous to a single testis from Drosophila or Nasonia. Within each follicle, developing sperm can be seen within cysts of various stages of development with the most immature stages toward one end and mature sperm toward the other end of the 


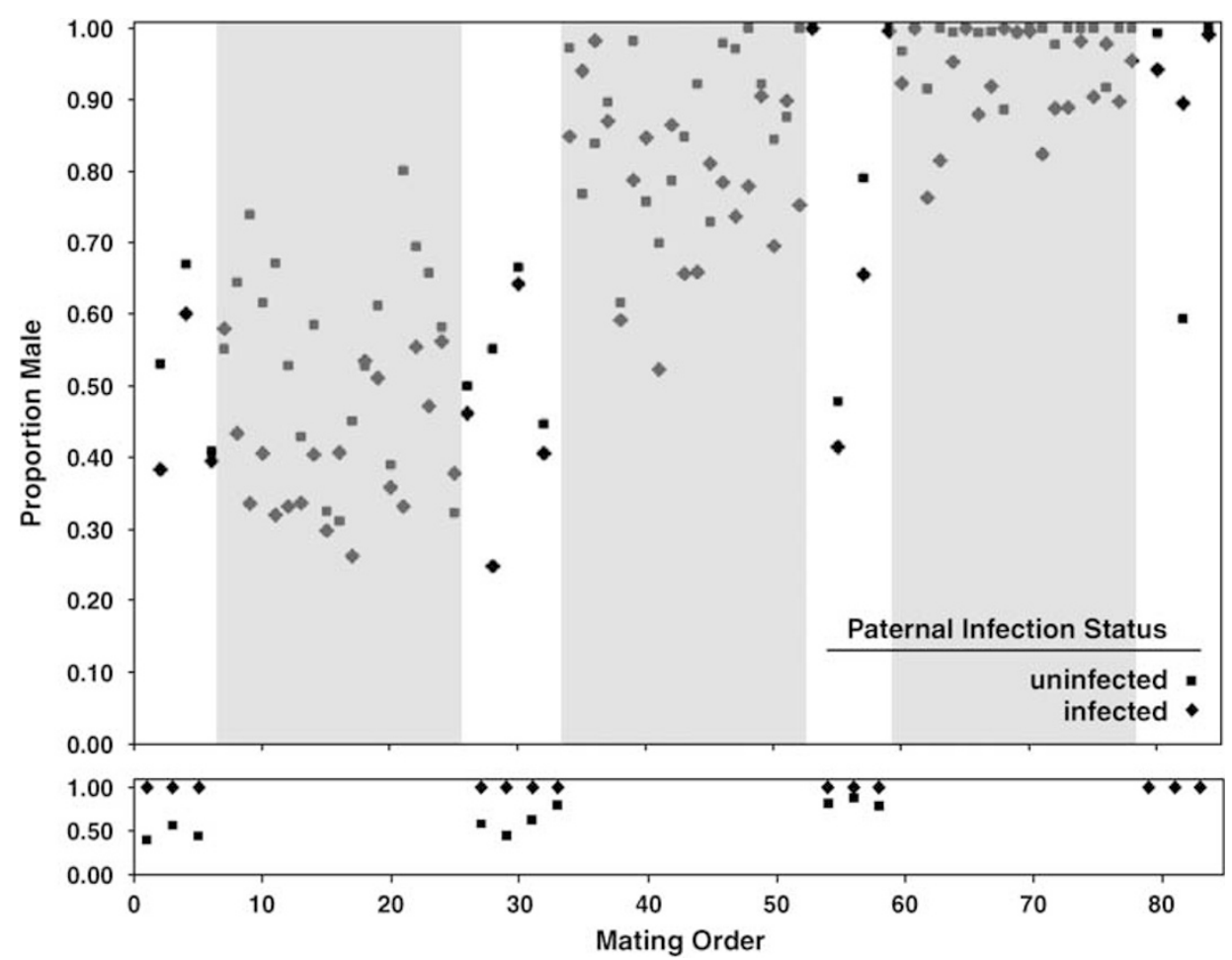

Figure 3 Sperm depletion, sex ratio and CI in N. vitripennis. Wolbachia-infected and uninfected males mated to Wolbachia-infected (top) and -uninfected (bottom) females. Matings within the shaded area are overnight mass matings with multiple females, and precise mating order within these time periods is unknown.

follicle. In contrast to Drosophila, Wolbachia were never seen within the testis follicle interior (that is, within cysts), but were abundant in the outer testis sheath (Figures $4 \mathrm{~b}-\mathrm{e}$ ). Therefore, Wolbachia are present neither in developing spermatocytes nor in the somatic cyst cells. Over 80 cysts were examined in detail after removal from testes of four different males and confirmed the absence of Wolbachia within these cysts.

Despite that Wolbachia are not present in cysts of C. alternans, the same males for which testes were examined showed $\sim 75.8$ and $67.2 \% \mathrm{CI}$ in the two lines examined. Therefore, we conclude that Wolbachia are not required within cysts (either spermatocytes and spermatids or somatic cyst cells) to affect sperm modification in this species.

\section{Discussion}

Wolbachia cause CI by the modification of sperm through as yet unknown mechanisms. Previous work on the distribution and proliferation of Wolbachia during spermatogenesis in Drosophila suggested that Wolbachia are required within developing spermatocytes/spermatids to cause modification (Clark et al., 2003). This was based on the observation that Wolbachia are abundant in spermatocytes of young males, and both proportion of infected cysts and CI level decline with age. The results of our work, however, caution against generalizing results from Drosophila to other taxa, and further suggest that the conclusion that Wolbachia are required in spermatocytes of Drosophila to affect CI needs to be reexamined. The positive correlation between CI level and the proportion of developing sperm infected with Wolbachia in Drosophila may be because Wolbachia only modify spermatids if infected, or alternately, both CI level and proportion of spermatids infected may both be dependent on Wolbachia densities earlier in development (spermatogonial stem cells, pole cells and so on) where modification is actually taking place. Recently Riparbelli et al. (2007) have described defects in sperm development within the testes of infected but not uninfected males. These defects, which include abnormal axoneme and mitochondria, were not restricted to infected cysts, suggesting that uninfected cysts can be affected by Wolbachia. What connection, if any, these sperm defects have on the expression of CI is currently unknown. (Riparbelli et al., 2007). Certainly, the data presented here shows that Wolbachia are not needed in developing sperm to cause sperm modification in insect species from two different insect orders.

These results suggest several alternative hypotheses for induction of sperm modification by Wolbachia. First, Wolbachia may produce a factor in sheath (Nasonia and Chelymorpha) or somatic cyst cells (Nasonia) that are passed across membranes into developing spermatids. For example, Wolbachia proteins could be passed from the sheath cells to the developing spermatids using a pathway similar to the one found in Heliothis virescens, where sheath cells synthesize proteins, which are imported by cyst cells by pinocytosis of testicular fluid and transfered to spermatids (Miller et al., 1990). Alternatively, Wolbachia within the somatic cyst cells (Nasonia) and/or sheath cells (Nasonia and Chelymorpha) could alter expression and synthesis of gene products from the host, thus changing the products exported to the developing spermatids, or by other inductive effects of these cells on developing spermatids. A third possibility is that Wolbachia affect modification very early 

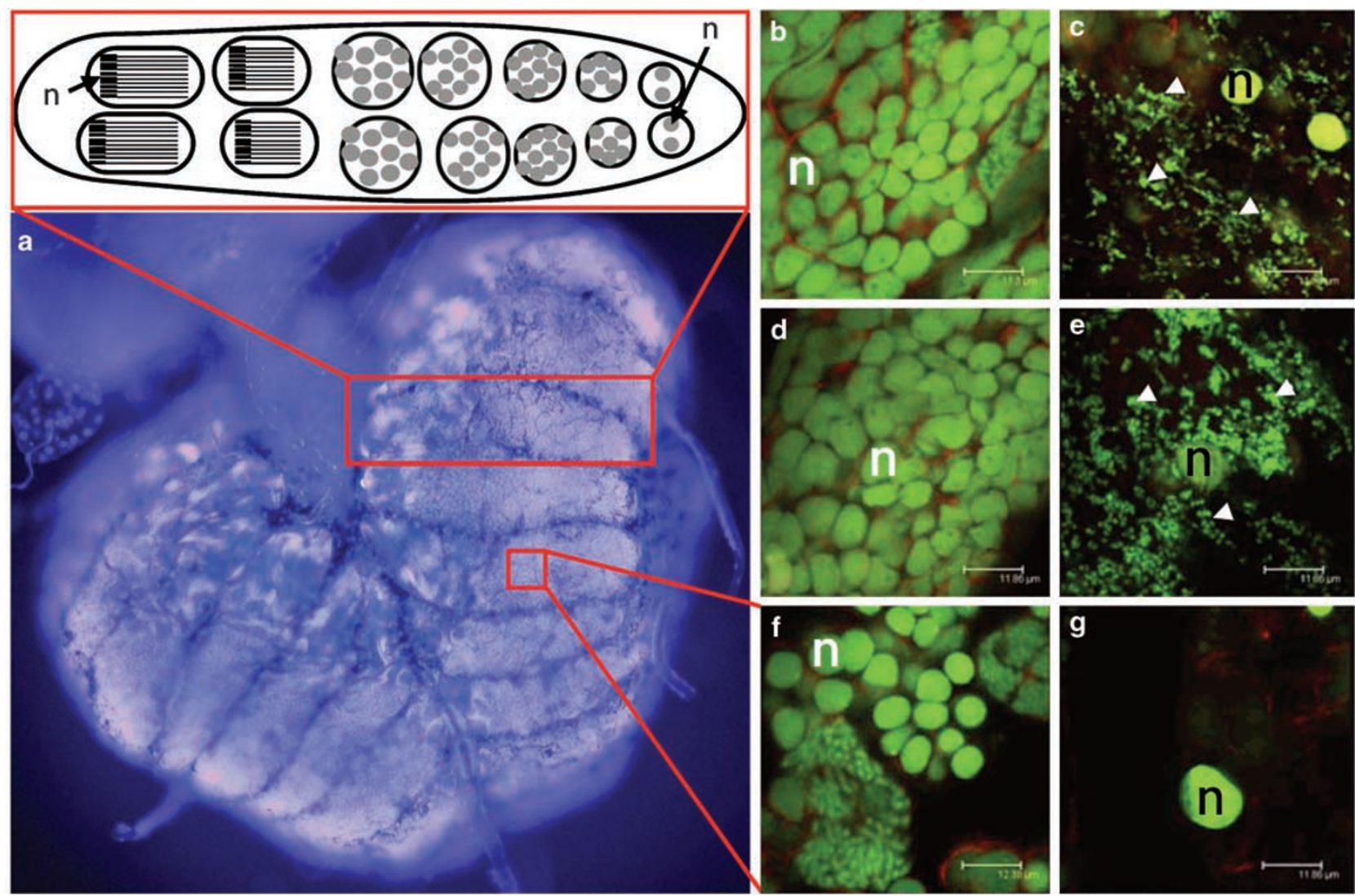

Figure 4 Spermatogenesis and Wolbachia in Chelymorpha alternans. (a) A pair of testes is shown for reference. The schematic (top) shows a single testis follicle with developing sperm cysts within. Development within the follicle outlined proceeds from right to left, with less developed cysts to the right and spermatids to the left. The square indicated approximate location of confocal sections (b-g). Wolbachia are not observed within spermatocytes (germline) from within a testis follicle from testes of 4-week-old (b) double- infected, (d) single-infected and (f) uninfected C. alternans. Wolbachia (arrow head) is observed only in the outer testis sheath, at the surface of the testes of 4-week-old (c) double-infected, (e) single-infected but not from (g) uninfected C. alternans. DNA is stained with DAPI (a) or Oligreen (b-g), host actin (red) is labeled with phalloidin $(\mathbf{b}-\mathbf{g}) . \mathrm{n}=$ host nuclei.

in the primordial germ cells, and Wolbachia are subsequently lost. For example, in Nasonia, Wolbachia are found in about $28 \%$ of spermatocytes. However, spermatocytes are connected by cytoplasmic bridges within individual cysts. Although difficult to quantify precisely, it appears that nearly all spermatocyte syncytia had at least one Wolbachia, suggesting that most primordial germ cells also had at least one Wolbachia cell. Consistent with $100 \%$ transmission of Wolbachia through the germline, an examination of pole cells in Nasonia embryos indicates that all or nearly all are infected with Wolbachia (data not shown). In the case of Chelymorpha, Wolbachia are totally absent from both spermatocytes/spermatids and somatic cyst cells in adult beetles. However, it is still possible that all pole cells were infected earlier in the development and Wolbachia were subsequently lost in germline daughter cells. Transmission of Wolbachia through the female germline is not complete in $C$. alternans (Keller et al., 2004), suggesting that not all pole cells were infected. Work in Drosophila has shown a positive correlation between the density of Wolbachia within pole cells and CI levels (Veneti et al., 2004). This may support Wolbachia-induced modification of pole cells or it may simply be a predictor of Wolbachia titer later in gemetogenesis when modification occurs. Modification could also occur within the spermatogonial stem cells within testes. Examination of sperm stem cells in
Nasonia and Chelymorpha would be challenging, as markers for the stem cell niche have not been described in either species.

What molecules, if any, Wolbachia are secreting into hosts is currently unknown. Wolbachia does possess a type IV secretion system, likely used for exporting molecules into host cells (Masui et al., 2000). It is currently known that Wolbachia produce bacteria phage, which can be found in developing sperm (Bordenstein et al., 2006). What effect Wolbachia phage has on host cells or the role in $\mathrm{CI}$ is currently unknown.

There is very little current data in any species concerning the timing of sperm modification during spermatogenesis. The only relevant experiment is in Drosophila simulans where heat-shock treatment of male third instar larvae results in a decrease in $\mathrm{CI}$; at this stage, the most mature sperm cysts typically are beginning spermatid elongation (Snook et al., 2000). The experiment may suggest that modification does not occur in embryonic stem cells, but other effects of heatshock on CI could also explain the results, and no apparent reductions in bacterial infection levels were observed. Clearly, more experiments on timing of sperm modification are needed.

In Drosophila, rates of CI have been shown to be dependent on a number of factors, including male age and male mating history (Turelli and Hoffmann, 1995; 
Clancy and Hoffmann, 1998; Karr et al., 1998; Reynolds et al., 2003). With subsequent matings, the rate of CI goes down dramatically in D. simulans (Karr et al., 1998). In $N$. vitripennis, there is no evidence of either a male age effect, or male mating history effect on CI (Figure 3). The male age and mating history effect in Drosophila could be explained by the depletion of Wolbachia from the male germline, likely spermatogonial stem cells (Clark et al., 2003). By this model, early developing sperm cysts contain Wolbachia and as a result are modified. With the depletion of Wolbachia, sperm cysts produced later in life lack Wolbachia and are not modified. Successive copulation of infected males depletes the older (modified) sperm and increases the proportion of newly matured (unmodified) sperm. Later copulations, therefore, have a higher proportion of unmodified sperm compared to similarly aged virgin males. The lack of decrease in CI in $N$. vitripennis with male age and successive copulation may either be due to the timing of spermatogenesis in Nasonia or due to fundamental differences in the modification of sperm by Wolbachia. In Nasonia, most of the lifetime sperm production is completed at or near the time of eclosion, before mating. (Pennypacker, 1958). Therefore, the population of sperm available at the first mating is the same as in subsequent matings.

As the molecular mechanism(s) resulting in Wolbachiainduced sperm modification remain elusive, different host taxa such as Nasonia and Chelymorpha provide excellent model systems to compliment studies in Drosophila. In Nasonia, we show that Wolbachia are able to modify a sperm, while not being present within an individual spermatocyte or spermatid during sperm development. In Chelymorpha, Wolbachia are able to modify sperm, while never being present in cysts.

\section{Acknowledgements}

This work was supported by the National Science Foundation FIBR Grant number EF-0328363.

\section{References}

Bailey-Jourdain C (2006). Male age effects, cytoplasmic incompatibilityy and the locallization of Wolbachia in Chelymorpha alternans Boh (Chrysomelidae, Cassidinae). McGill University: Montreal.

Bandi C, Anderson TJ, Genchi C, Blaxter ML (1998). Phylogeny of Wolbachia in filarial nematodes. Proc R Soc Lond B Biol Sci 265: 2407-2413.

Bordenstein SR, Marshall ML, Fry AJ, Kim U, Wernegreen JJ (2006). The tripartite associations between bacteriophage, Wolbachia, and arthropods. PLoS Pathogens 2: e43.

Bordenstein SR, Uy JJ, Werren JH (2003). Host genotype determines cytoplasmic incompatibility type in the haplodiploid genus nasonia. Genetics 164: 223-233.

Bordenstein SR, Werren JH (1998). Effects of A and B Wolbachia and host genotype on interspecies cytoplasmic incompatibility in Nasonia. Genetics 148: 1833-1844.

Breeuwer JA, Jacobs G (1996). Wolbachia: intracellular manipulators of mite reproduction. Exp Appl Acarol 20: 421-434.

Breeuwer JA, Werren JH (1990). Microorganisms associated with chromosome destruction and reproductive isolation between two insect species. Nature 346: 558-560.

Bressac C, Rousset F (1993). The reproductive incompatibility system in Drosophila simulans: DAPI-staining analysis of the Wolbachia symbionts in sperm cysts. J Invertebr Pathol 61: 226-230.
Brower JH (1976). Cytoplasmic incompatibility: occurence in a stored-product pest Ephestia cautella. Ann Entomol Soc Am 69: 1011-1015.

Callaini G, Riparbelli MG, Giordano R, Dallai R (1996). Mitotic defects associated with cytoplasmic incompatibility in Drosophila simulans. I Invertebr Pathol 67: 55-64.

Caspari E, Watson GS (1959). On the evolutionary importance of cytoplasmic sterility in mosquitoes. Evolution 13: 568-570.

Clancy DJ, Hoffmann AA (1998). Environmental effects on cytoplasmic incompatibility and bacterial load in Wolbachiainfected Drosophila simulans. Entomologia Experimentalis et Applicata 86: 13-24.

Clark ME (2007). Wolbachia Symbiosis in Arthropods. In: Hoerauf A, Rao R (eds). Wolbachia: A Bug's Life in Another Bug. Karger: Basel, pp 90-123.

Clark ME, Veneti Z, Bourtzis K, Karr TL (2002). The distribution and proliferation of the intracellular bacteria Wolbachia during spermatogenesis in Drosophila. Mech Dev 111: 3-15.

Clark ME, Veneti Z, Bourtzis K, Karr TL (2003). Wolbachia distribution and cytoplasmic incompatibility during sperm development: the cyst as the basic cellular unit of $\mathrm{CI}$ expression. Mech Dev 120: 185-198.

Fuller MT (1993). Spermatogenesis. In: Bates $M$ and Martinez Arrias A (ed). The Development of Drosophila melanogaster. Cold Spring Harbor Press, Cold Spring Harbor: NY, pp 71-147.

Hilgenboecker K, Hammerstein P, Schlattmann P, Telschow A, Werren JH (2008). How many species are infected with Wolbachia?-A statistical analysis of current data. FEMS Microbiol Letters 281: 215-220.

Hoshizaki S, Shimada T (1995). PCR-based detection of Wolbachia, cytoplasmic incompatibility microorganisms, infected in natural populations of Laodelphax striatellus (Homoptera: Delphacidae) in central Japan: has the distribution of Wolbachia spread recently? Insect Mol Biol 4: 237-243.

Jeyaprakash A, Hoy MA (2000). Long PCR improves Wolbachia DNA amplification: wsp sequences found in $76 \%$ of sixtythree arthropod species. Insect Mol Biol 9: 393-405.

Kamoda S, Masui S, Ishikawa H, Sasaki T (2000). Wolbachia infection and cytoplasmic incompatibility in the cricket Teleogryllus taiwanemma. J Exp Biol 203 (Pt 16): 2503-2509.

Karr TL, Yang W, Feder ME (1998). Overcoming cytoplasmic incompatibility in Drosophila. Proc R Soc Lond B Biol Sci 265: 391-395.

Keller GP, Windsor DM, Saucedo JM, Werren JH (2004). Reproductive effects and geographical distributions of two Wolbachia strains infecting the Neotropical beetle, Chelymorpha alternans Boh. (Chrysomelidae, Cassidinae). Mol Ecol 13: 2405-2420.

Masui S, Sasaki T, Ishikawa H (2000). Genes for the type IV secretion system in an intracellular symbiont, Wolbachia, a causative agent of various sexual alterations in arthropods. J Bacteriol 182: 6529-6531.

Miller SG, Leclerc RF, Seo S-J, Malone C (1990). Synthesis and transport of storage proteins by testes in Heliothis virescens. Arch Insect Biochem Physiol 14: 151-170.

Moret Y, Juchault P, Rigaud T (2001). Wolbachia endosymbiont responsible for cytoplasmic incompatibility in a terrestrial crustacean: effects in natural and foreign hosts. Heredity $\mathbf{8 6}$ 325-332.

Pennypacker MI (1958). The chromosomes of the parasitic wasp, Mormoniella vitripennis. I. In spermatogenesis of haploid and diploid males. Arch Biol 69: 483-495.

Perrot-Minnot MJ, Guo LR, Werren JH (1996). Single and double infections with Wolbachia in the parasitic wasp Nasonia vitripennis: effects on compatibility. Genetics 143: 961-972.

Poinsot D, Bourtzis K, Markakis G, Savakis C, Mercot H (1998). Wolbachia transfer from Drosophila melanogaster into $D$. simulans: Host effect and cytoplasmic incompatibility relationships. Genetics 150: 227-237. 
Pultz MA, Leaf DS (2003). The jewel wasp Nasonia: querying the genome with haplo-diploid genetics. Genesis 35: 185-191.

Reed KM, Werren JH (1995). Induction of paternal genome loss by the paternal-sex-ratio chromosome and cytoplasmic incompatibility bacteria (Wolbachia): a comparative study of early embryonic events. Mol Reprod Dev 40: 408-418.

Reynolds KT, Thomson LJ, Hoffmann AA (2003). The effects of host age, host nuclear background and temperature on phenotypic effects of the virulent Wolbachia strain popcorn in Drosophila melanogaster. Genetics 164: 1027-1034.

Riparbelli MG, Giordano R, Callaini G (2007). Effects of Wolbachia on sperm maturation and architecture in Drosophila simulans Riverside. Mech Dev 124: 699-714.

Sintupachee S, Milne JR, Poonchaisri S, Baimai V, Kittayapong P (2006). Closely related Wolbachia strains within the pumpkin arthropod community and potential for horizontal transmission via the plant. Microb Ecol 51: 294-301.

Snook RR, Cleland SY, Wolfner MF, Karr TL (2000). Offsetting effects of Wolbachia infection and heat shock on sperm production in Drosophila simulans: analyses of fecundity, fertility and accessory gland proteins. Genetics 155: 167-178.

Stouthamer R, Breeuwer JA, Hurst GD (1999). Wolbachia pipientis: microbial manipulator of arthropod reproduction. Annu Rev Microbiol 53: 71-102.

Tram U, Fredrick K, Werren JH, Sullivan W (2006). Paternal Chromosome segregation during the first mitotic division determines cytoplasmic incompatibility phenotype. J Cell Science 119: 3655-3663.

Turelli M, Hoffmann AA (1995). Cytoplasmic incompatibility in Drosophila simulans: dynamics and parameter estimates from natural populations. Genetics 140: 1319-1338.
Veneti Z, Clark ME, Karr TL, Savakis C, Bourtzis K (2004). Heads or tails: host-parasite interactions in the Drosophila-Wolbachia system. Appl Environ Microbiol 70: 5366-5372.

Veneti Z, Clark ME, Zabalou S, Karr TL, Savakis C, Bourtzis K (2003). Cytoplasmic incompatibility and sperm cyst infection in different Drosophila-Wolbachia associations. Genetics 164: 545-552.

Wade MJ, Stevens L (1985). Microorganism mediated reproductive isolation in flour beetles (genus Tribolium). Science 227: 527-528.

Werren JH (1997). Biology of Wolbachia. Annu Rev Entomol 42: 587-609.

Werren JH, O'Neill S (1997). The evolution of heritable symbionts. In: O'Neil S, Hoffmann AA, Werren JH (eds). Influential Passengers: Inherited Microorganisms and Arthropod Reproduction. Oxford University Press: New York, pp 1-41.

Werren JH, Windsor D, Guo L (1995). Distribution of Wolbachia among Neotropical arthropods. Proc R Soc Lond B Biol Sci 262: 197-204.

West SA, Cook JM, Werren JH, Godfray HC (1998). Wolbachia in two insect host-parasitoid communities. Mol Ecol 7: $1457-1465$.

Whiting AR (1967). The Biology of the Parasitic Wasp Mormoniella vitripennis [ = Nasonia brevicornis] (Walker). Q Rev Biol 42: 333-406.

Windsor D, Werren JH (2000). Wolbachia infection frequencies in insects: evidence of a global equilibrium. Proc $R$ Soc Lond $B$ Biol Sci 267: 1277-1285.

Yen JH (1975). Transovarial transmission of Rickettsia-like microorganisms in mosquitoes. Ann N Y Acad Sci 266: 152-161. 